\title{
Enigmatic photon absorption in plasmas near solar interior conditions
}

\author{
CARlos A. Iglesias \\ Lawrence Livermore National Laboratories \\ P.O. Box 808, Livermore, CA 94550, USA
}

\begin{abstract}
Large systematic discrepancies between theoretical and experimental photon absorption of Fe plasmas applicable to the solar interior were reported [Bailey et al., Nature 517, 56(2015)]. The disagreement is examined in the context of the Thomas-Reiche-Kuhn f-sum rule. The analysis identifies several anomalies in the experimental results.
\end{abstract}

Keywords:

Opacity, f-sum rule, solar interior

Corresponding author:

E-mail: iglesias1@1lnl.gov (C. A. Iglesias) 


\section{Introduction}

Plasma radiative properties are integral to the study of astrophysics, inertial and magnetic confinement, and warm dense matter. The opacity calculations for these problems, however, entail approximations to myriad atomic processes. Furthermore, models for fundamental phenomena, such as continuum lowering and spectral line broadening, remain mostly untested. Hence, there is interest in assessing theoretical opacities by comparisons to photon absorption measurements. In response, experiments were done at the Sandia National Laboratories Zmachine to measure the photon transmission of plasmas at conditions applicable to the solar interior. An important goal of the experiments was to address discrepancies between helioseismic observations and solar models, which can be reconciled by $\sim 15 \%$ increases in the opacity near the solar radiation-convection boundary [1].

Although models agreed well with earlier measurements [2], there are significant systematic discrepancies with more recent data at higher densities and temperatures [3]. As an example, Fig. 1 compares the Fe experimental absorption and the effective opacity (see Appendix) from the Topaz code [4]. The disagreement is not unique to Topaz and other models yield similar discrepancies [3]. The disagreement in Fig. 1 is examined in context with the Thomas-ReicheKuhn f-sum rule [5]. This sum rule follows from general principles and constrains the sum of oscillator strengths. The analysis identifies several anomalies in the recent Sandia data [3] absent in the earlier measurements [2].

\section{Analysis and discussion}

The spectrum in Fig. 1 is predominantly absorption by L-shell electrons in Fe. The Sandia data show $2 \mathrm{p}-4 \mathrm{~d}$ absorption lines from Ne-like $(\lambda \approx 12.2 \AA)$, F-like $(\lambda \approx 11.4 \AA$ ), and O-like Fe $(\lambda \approx 10.6 \AA)$ [3]. The Topaz calculation assumes local thermal equilibrium and yields an average ionization charge $\langle Z\rangle=16.9$ for Fe and displays similar $2 \mathrm{p}-4 \mathrm{~d}$ lines. Furthermore, there is good correspondence, except for overall strength, between the spectral lines in the data and the model suggesting reasonable agreement in the ion charge distribution.

A possible error in the opacity models is the calculation of photon absorption cross-sections by L-shell electrons. As a test, Fig. 2 presents the Topaz photon ionization cross-section for the ground configuration of atomic Cr-like Fe in good agreement with the cold solid density data, $\sigma_{\text {cold }}[6]$. The figure also presents calculations for ground configurations (single-configuration 
approximation without term structure) for several other Fe ions. These calculations reproduce the known result that removing $\mathrm{M}$-shell electrons increases the ionization threshold without further impacting the cross-section [7]. It is only when L-shell electrons are removed that the crosssection, which is proportional to the number of L-shell electrons, is reduced. Although not explicitly displayed here, these results extend to configurations with excited electrons. For example, the cross-section for Ne-like Fe with one excited electron is similar (except for threshold energy) to the cross-section for the ground configuration of F-like Fe.

The absorption in solids at photon energies above $\sim 50 \mathrm{eV}$ (avoids valence orbitals or bands) are atomic-like except near thresholds where features associated with the solid state can appear [8]. In plasmas, there is not only continuum lowering [9] but there are fluctuations [10] that smooth the absorption at energies below the isolated ionization threshold. The smoothing conserves the oscillator strength density, $d f / d \varepsilon$, where $f$ and $\varepsilon$ are the oscillator strength and photon energy, respectively, of the bound-bound transitions in the isolated ion [11-13].

To proceed, consider the f-sum rule [5],

$$
\sum_{\beta} f_{\alpha \beta}=N
$$

This equation applies provided relativistic effects are small (valid for Fe [14]) and refers to an atomic system containing $N$ bound electrons and the oscillator strength sum for transitions from state $\alpha$ to all final states $\beta$ including the continuum. Although full configuration interactions (CI) effects do not impact the sum rule in Eq. (2), if they are negligible or neglected then Eq. (2) extends to partial sums over subshells [5],

$$
\sum_{n^{\prime} \ell^{\prime}} f_{n \ell, n^{\prime} \ell^{\prime}}=N_{n \ell}
$$

where $N_{n \ell}$ is the $n \ell$ subshell electron occupation ( $n$ and $\ell$ are principal and orbital quantum numbers). Since the Topaz code neglects full CI [15] and excited electronic configurations are delocalized in solid Fe, both the Topaz calculations and the cold data satisfy Eq. (3).

As the examples in Figs. 2 show, the Topaz cross-sections above the ionization threshold are similar to $\sigma_{\text {cold }}$ for Fe ions with full L-shell configurations. Accordingly, the bound-bound contributions should reproduce the remaining portion of the sum in Eq. (3). For partially filled Lshell ions the sum in Eq. (3) is proportionally smaller than for $\sigma_{\text {cold }}$. Therefore, recalling that the 
oscillator strength density is conserved [11-13], a smoothed cross-section obtained by the following convolution is constrained to have $\sigma_{\text {cold }}$ as a maximum,

$$
\begin{aligned}
K(\lambda) & =\int_{-\infty}^{\infty} d \lambda^{\prime} G\left(\lambda-\lambda^{\prime}\right) \kappa\left(\lambda^{\prime}\right) \\
& \leq \sigma_{\text {cold }}(\lambda)
\end{aligned}
$$

where $\kappa(\lambda)$ is the absorption cross-section at photon wavelength $\lambda$ and $G(\lambda)$ is an arbitrary, normalized response function with variance sufficiently large to smear features in the spectrum. The idea is illustrated in Fig. 3 using a Gaussian response function where the photon absorption from ground configurations of $\mathrm{C}$ - and $\mathrm{Mg}$-like $\mathrm{Fe}$ are compared to $\sigma_{\text {cold }}$. These Topaz calculations assumed the same electron temperature and density as Fig. 1 for the plasma effects and used intermediate coupling for the configuration term structure.

Plots of $K(\lambda)$ are displayed in Fig. 4 for the recent Sandia data [3] and Topaz opacity (not the Topaz effective opacity since it artificially alters the value of $K(\lambda)$, see Fig. 5). Clearly, the Sandia data exceed $\sigma_{\text {cold }}$ at lower wavelengths and differ in slope. The Topaz result remains below the cold data as expected since on average it has a partially filled L-shell.

It is instructive to integrate $K(\lambda)$ over the range of the data $(\lambda=8-13 \AA)$. The Topaz calculations and Sandia data yield $72 \%$ and $108 \%$, respectively, relative to the integration over the cold data. The Topaz integration, with an average of 6.04 electrons in the L-shell (on average 1.68 and 4.36 in the $2 \mathrm{~s}$ and $2 \mathrm{p}$ subshells, respectively), is consistent with the full L-shell of the cold data. On the other hand, the Sandia and cold data integrations essentially agree. The agreement suggests a full L-shell for the Sandia data in conflict with the observed strong spectral lines from open L-shell ions. The difference in slope does not necessarily imply a violation of the f-sum rule, but requires a modification in oscillator strength density relative to the cold data.

A conjecture to reconcile the discrepancies in Fig. 1 is missing transitions in the calculations [3]. One possibility is models neglecting low probability electronic configurations, which when combined make a significant contribution to the spectrum. In the Topaz calculations, which satisfy the f-sum rule as well as conserve $d f / d \varepsilon$ and particle number, such missing transitions may fill in gaps and weaken existing spectral features (in effect broadening the lines), but cannot increase $K(\lambda)$ above the cold data or change the overall slope. 
Another possibility is a redistribution of $d f / d \varepsilon$ unaccounted by the models. It is unlikely for the shift in oscillator strength to come at the expense of the photon ionization cross-section at high energies not measured in the experiments. The high-energy photon ionization is expected to be atomic-like [8] and well reproduced by the models. The lower energy $n=2$ to 3 lines, which are outside the experimental spectral range, are relatively strong (see Fig. 5) so a transfer of strength from these lines to higher energies could reduce the discrepancies. The well-defined $n=2$ to 4 spectral lines in the measured spectra, however, imply that the $n=2$ to 3 transitions involve deeply bound orbitals. That is, many of these transitions occur in excited electronic configurations [3] that can impact spectral line shapes, but the line strength is proportional to the dipole radial integral involving the $\mathrm{n}=2$ and 3 atomic-like wavefunctions [5].

A full CI treatment, not performed in the Topaz calculations [15], may transfer oscillator strength to higher energies [5]. The earlier measurements, however, involved similar charge states [2,3] and models without full CI show good agreement. In particular, there is excellent agreement at lower wavelengths (see Fig. 6). Furthermore, calculations from the Opacity Project [16], which include full CI effects, also compare poorly with the data (see Fig. 3 in Ref. 3).

\section{Conclusion}

The disagreement between opacity models and the recent Sandia data [3] is enigmatic. The models reproduce the colder and lower density Sandia data [2] as well as the cold, solid density measurements [6]. The latter has higher free electron densities than the plasmas in the Sandia experiments, but not the excited level populations associated with high temperatures. On the other hand, the Sandia experiments include supporting measurements to test the reliability of the data $[2,3,17,18]$.

The present analysis, however, identified anomalies in the recent Sandia data [3]. Specifically, the recent Sandia data [3] exceed the cold, solid density absorption [6] at low photon wavelengths. This enhanced absorption seems irresoluble with theory since calculations satisfy the f-sum rule as well as conserve oscillator strength density and particle number. That is, the largest theoretical opacity is obtained from ions with a full L-shell, which reproduces the cold data at small wavelengths. The analysis revealed other puzzling aspects of the data: the integrated absorption suggests a full L-shell inconsistent with the measured spectrum, which displays strong spectral lines from F- and O-like configurations, and the average slope of the 
absorption disagrees with that of the cold data. In addition, the older Sandia data [2], which are in good agreement with theoretical calculations, were shown consistent with the f-sum rule and the cold, solid density absorption cross-section.

Finally, the Sandia experiments [2,3] indicate, in the absence of unknown systematic experimental errors, that for certain matter conditions photon absorption by plasmas can appreciably redistribute the oscillator strength density relative to calculations. This phenomenon has not been previously observed and independent measurements are welcome to corroborate these extraordinary results. The future experiments should consider a wider spectral range to help quantify any redistribution of oscillator strength density.

\section{Acknowledgments}

It is a pleasure to thank J.E. Bailey and J.P. Colgan for valuable discussions. Special thanks are due to J.E. Bailey for the Sandia data prior to publication. This work performed under the auspices of the U.S. Department of Energy by Lawrence Livermore National Laboratory under Contract DE-AC52-07NA27344. 


\section{Appendix. Comparison of theoretical and experimental opacities}

To account for finite spectral resolution, the opacity calculations are not directly compared to the extracted experimental absorption. Instead, a theoretical transmission is first computed using experimental parameters, which is convolved with the instrumental function. So that the effective opacity for comparison to the data is obtained by

$$
\tilde{\kappa}(\lambda)=-\left(\frac{1}{\rho L}\right) \ln \left\{\int_{-\infty}^{\infty} d \lambda^{\prime} F_{I n s}\left(\lambda-\lambda^{\prime}\right) e^{-\rho L \kappa\left(\lambda^{\prime}\right)}\right\}
$$

with $\kappa(\lambda)$ the model opacity at photon wavelength $\lambda, \rho L=9.3 \times 10^{-5} \mathrm{~g} / \mathrm{cm}^{2}$ the Fe areal density, and $F_{\text {Ins }}(\lambda)$ the instrument function with resolving power $\lambda / \delta \lambda=700$ [3] assumed a Gaussian in the present calculations. The procedure suppresses the range of absorption compared to $\kappa(\lambda)$. The effects are displayed in Fig. 5 indicating that finite spectral resolution $[19,20]$ is unlikely to explain the discrepancies in Fig. 1. Specifically, there is negligible effect for $\lambda \leq 10 \AA$, otherwise the minima (maxima) are increased (decreased) by $10-20 \%$ except for features at $\lambda \geq 13 \AA$ beyond the experimental spectrum where line peaks are reduced by as much as a factor of 6 . The large wavelength region, however, may require second-order crystal reflections and strong absorption lines can be complicated by self-emission [3].

There was no comparison of the Topaz calculations to the earlier Sandia data [2], which is now displayed in Fig. 6 showing good agreement. The experimental spectrum in Fig. 6 is generated from several measurements using thin and thick samples introducing shot-to-shot variations. There is a noticeable disagreement, but this occurs near the seam $(\sim 12.5 \AA)$ where thin and thick samples were joined [2]. Also included in the figure is the recent Sandia data [3], but note that the $\mathrm{Mg}$ diagnostic lines [2,3] at $\lambda=8.4 \AA$ and $9.2 \AA$ were removed [21]. It shows that similar ionization states were sampled in the different measurements and the recent Sandia data has an overall enhanced opacity.

For comparison, the Fe cold solid density absorption [6] is displayed in Fig. 7 together with $K(\lambda)$ in Eq. (1) for the earlier Sandia experiments. Although the older Sandia data [2] could not be further smeared without introducing significant boundary effects, it clearly does not exceed the cold, solid density cross-section. As before, the Topaz smoothed opacity, which on average has a partially filled L-shell, remains below the cold data. 


\section{References}

[1] S. Basu H.M. \& Antia, Physics Reports 457, 217 (2008)

[2] J.E. Bailey, G.A. Rochau, C.A. Iglesias et al., Phys.Rev.Lett. 99, 265002(2007)

[3] J.E. Bailey, T. Nagayama, G.P. Loisel et al., Nature 517, 56(2015)

[4] C.A. Iglesias, M.H. Chen, V. Sonnad et al., JQSRT 81, 227 (2003)

[5] R. Cowan, The Theory of Atomic Structure (California Press, Berkeley, 1974)

[6] B.L. Henke, E.M. Gullikson \& J.C. Davis, Atomic Data \& Nuclear Data Tables 54, 181(1993); http://henke.lbl.gov/optical_constants/

[7] G. Nasreen, S.T. Manson \& P.C. Deshmukh, Phys.Rev. A40, 6091(1989)

[8] B.L. Henke, P. Lee, T.J. Tanaka et al., Atomic Data \& Nuclear Data Tables, 27, 1(1982)

[9] J.C. Stewart \& K.D. Pyatt, Ap.J. 144,1203(1966)

[10] D.R. Inglis \& E. Teller, Ap.J. 90, 439(1939)

[11] F.E. Höhne \& R. Zimmermann, J.Phys. B15, 2551(1982)

[12] R.M. More, Adv. At. Mol. Phys. 21, 305(1985)

[13] E.J. Iglesias \& H.R. Griem, JQSRT 55, 383(1996)

[14] For example, relativistic corrections to atomic scattering factor calculations using the f-sum rule are proportional to $\left(h v / m c^{2}\right)^{2}$ with $h v$ the photon energy and $m c^{2}$ the electron rest mass energy, which are negligible for $\lambda>6 \AA$ (experimental spectral region) [8].

[15] The Topaz calculations except for those in Fig. 2 used intermediate angular momentum coupling [4,5]; thus, include CI between spin-orbit sub-arrays belonging to the same nonrelativistic configuration.

[16] N.R. Badnell, M.A. Bautista, K. Butler et al., MNRAS 360, 458(2005)

[17] T. Nagayama, J.E. Bailey, G.A. Rochau et al., Rev.Sci.Instrum. 83, 10E128(2012)

[18] T. Nagayama, J.E. Bailey, G. Loisel et al., Phys.Plas. 21, 056502(2014)

[19] S.J. Davidson, C.S. Lewis, D. O’Neil et al., Laser Interaction with Matter, eds. G. Velarde, E. Minguez \& J.M. Peraldo (World Scientific, Singapore, 1989)

[20] C.A. Iglesias, JQSRT 99, 295 (2006)

[21] J.E. Bailey, private communication 


\section{Figure Captions}

Fig 1 - Comparison of experimental and theoretical Fe photon absorption cross-section as a function of wavelength at electron temperature and density of $182 \mathrm{eV}$ and $3.1 \times 10^{22} \mathrm{~cm}^{-3}$ : Sandia data [3] (black) and TOPAZ effective opacity (grey).

Fig. 2 - Comparison of the Fe cold absorption cross-section as a function of wavelength and Topaz calculations for ground configurations of various Fe ions indicated in the figure.

Fig. 3 - The Topaz photon absorption cross-section as function of wavelength for the ground configuration of C- and Mg-like Fe (grey) and smoothed using Eq. (1) (black) are compared to $\sigma_{\text {cold }}($ dots $)$. The C-like results are scaled by 0.01 for clarity.

Fig 4 - The smoothed Fe photon absorption, $K(\lambda)$, as a function of wavelength at conditions of Fig. 1 for the Sandia data [3] (solid) and Topaz (dash) are compared to $\sigma_{\text {cold }}(\lambda)($ dots $)$.

Fig 5-Comparison of the Topaz Fe opacity (black) and effective opacity (grey) as a function of wavelength at conditions of Fig. 1.

Fig 6 - Comparison of experimental and theoretical Fe photon absorption cross-section as a function of wavelength at a temperature and electron density of $156 \mathrm{eV}$ and $6.9 \times 10^{21} \mathrm{~cm}^{-}$

3: Sandia data [2] (thin black) and Topaz effective opacity (grey). Also included is the recent Sandia data [3] (thick black) at electron temperature and density of $182 \mathrm{eV}$ and $3.1 \times 10^{22} \mathrm{~cm}^{-3}$.

Fig 7 - The smoothed Fe photon absorption, $K(\lambda)$, as a function of wavelength at conditions of Fig. 6 for the Sandia data [2] (solid) and Topaz (dash) are compared to $\sigma_{\text {cold }}(\lambda)($ dots $)$. 


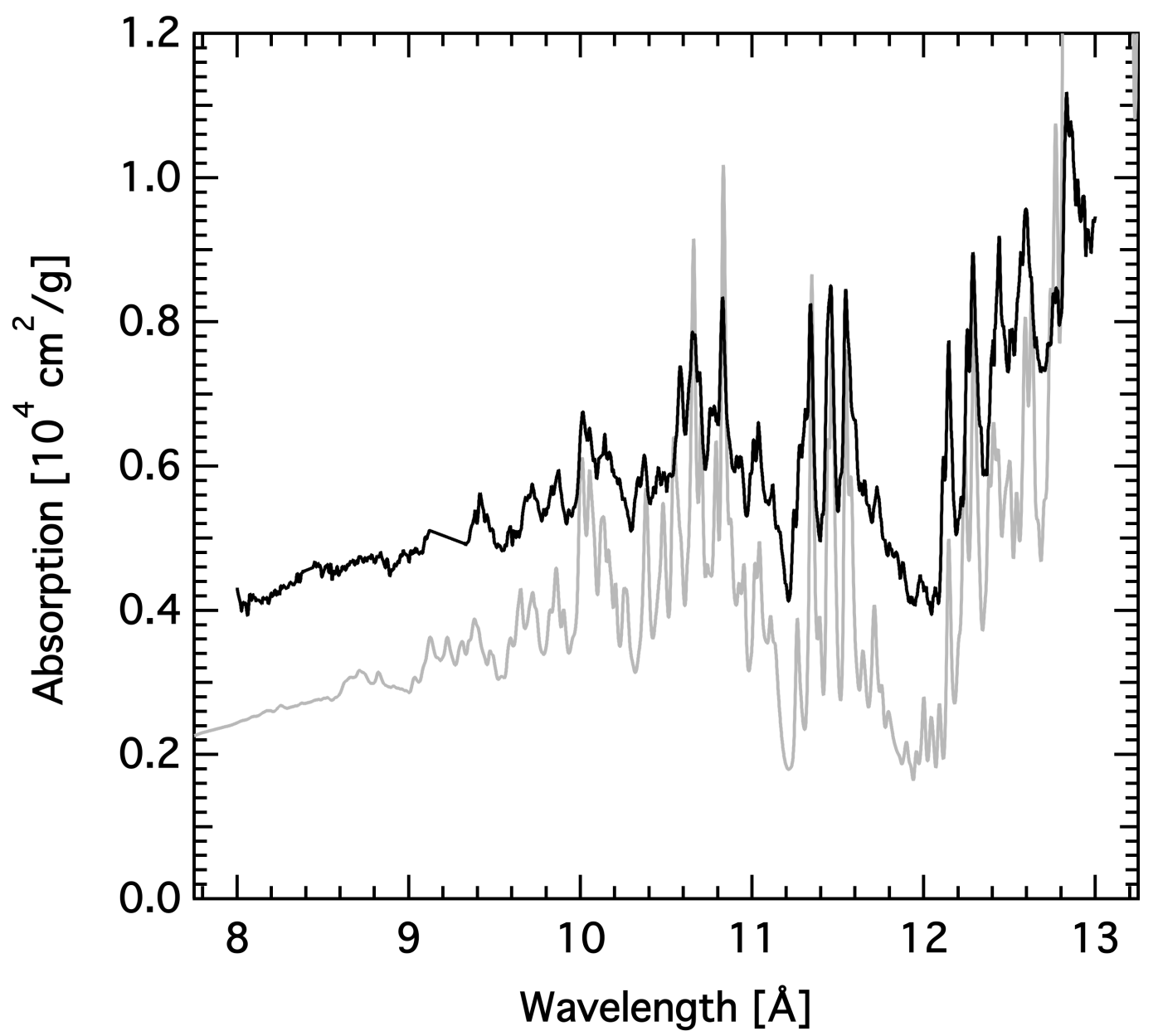

Fig 1 - Comparison of experimental and theoretical Fe photon absorption cross-section as a function of wavelength at electron temperature and density of $182 \mathrm{eV}$ and $3.1 \times 10^{22} \mathrm{~cm}^{-3}$ : Sandia data [3] (black) and TOPAZ effective opacity (grey). 


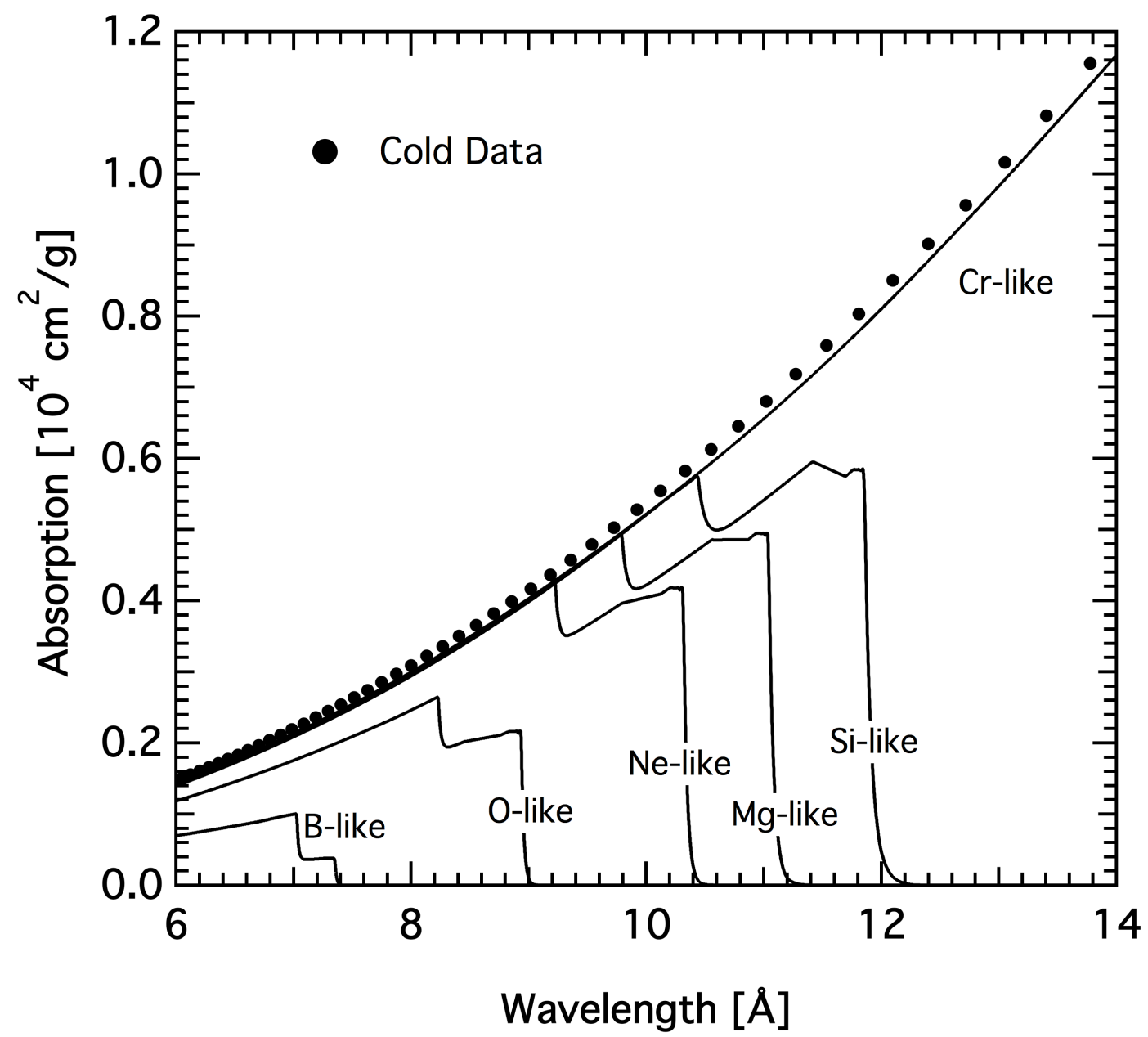

Fig. 2 - Comparison of the Fe cold absorption cross-section as a function of wavelength and Topaz calculations for ground configurations of various Fe ions indicated in the figure. 


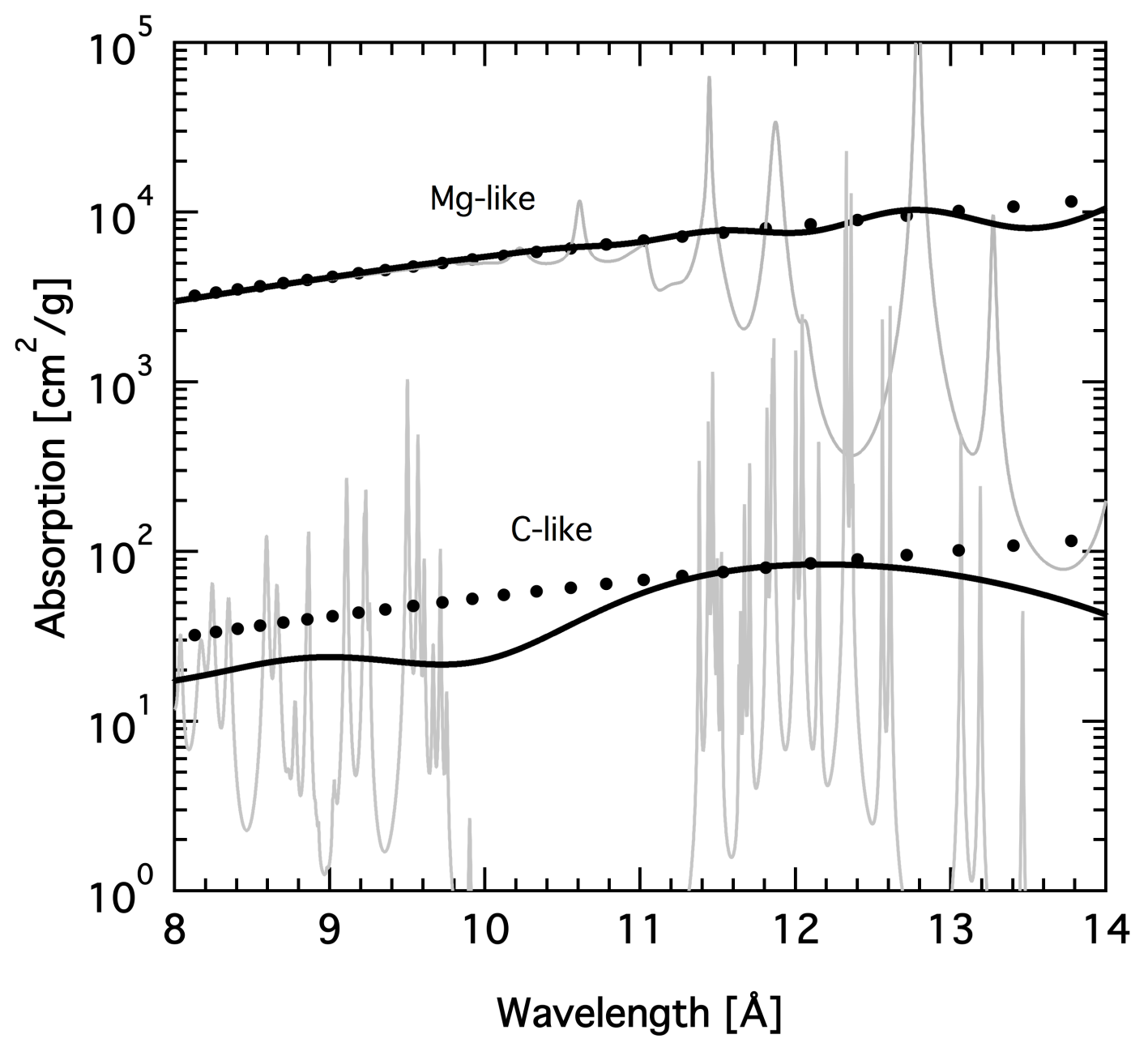

Fig. 3 - The Topaz photon absorption cross-section as function of wavelength for the ground configuration of C- and Mg-like Fe (grey) and smoothed using Eq. (1) (black) are compared to $\sigma_{\text {cold }}($ dots $)$. The C-like results are scaled by 0.01 for clarity. 


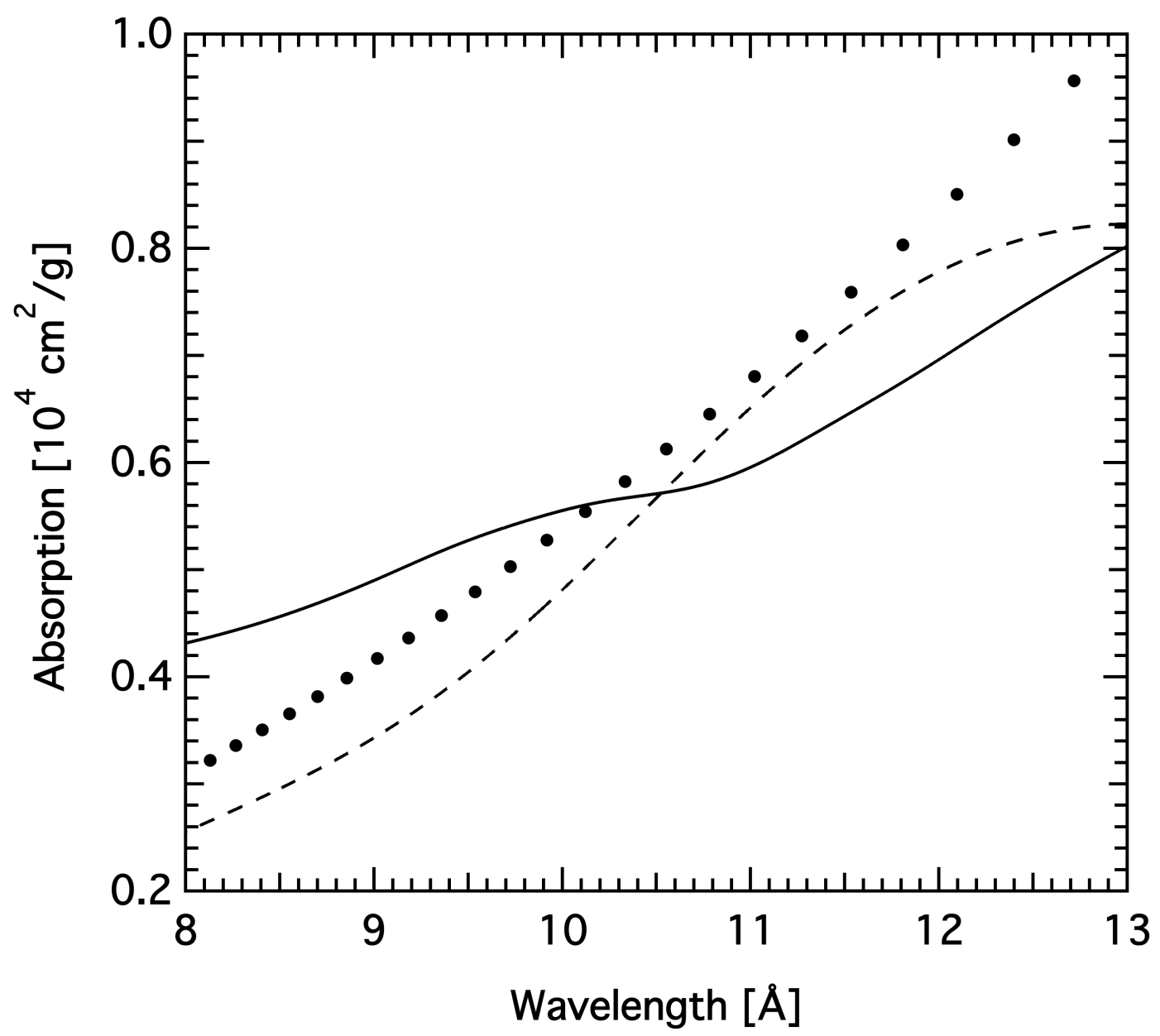

Fig 4 - The smoothed Fe photon absorption, $K(\lambda)$, as a function of wavelength at conditions of Fig. 1 for the Sandia data [3] (solid) and Topaz (dash) are compared to $\sigma_{\text {cold }}(\lambda)($ dots). 


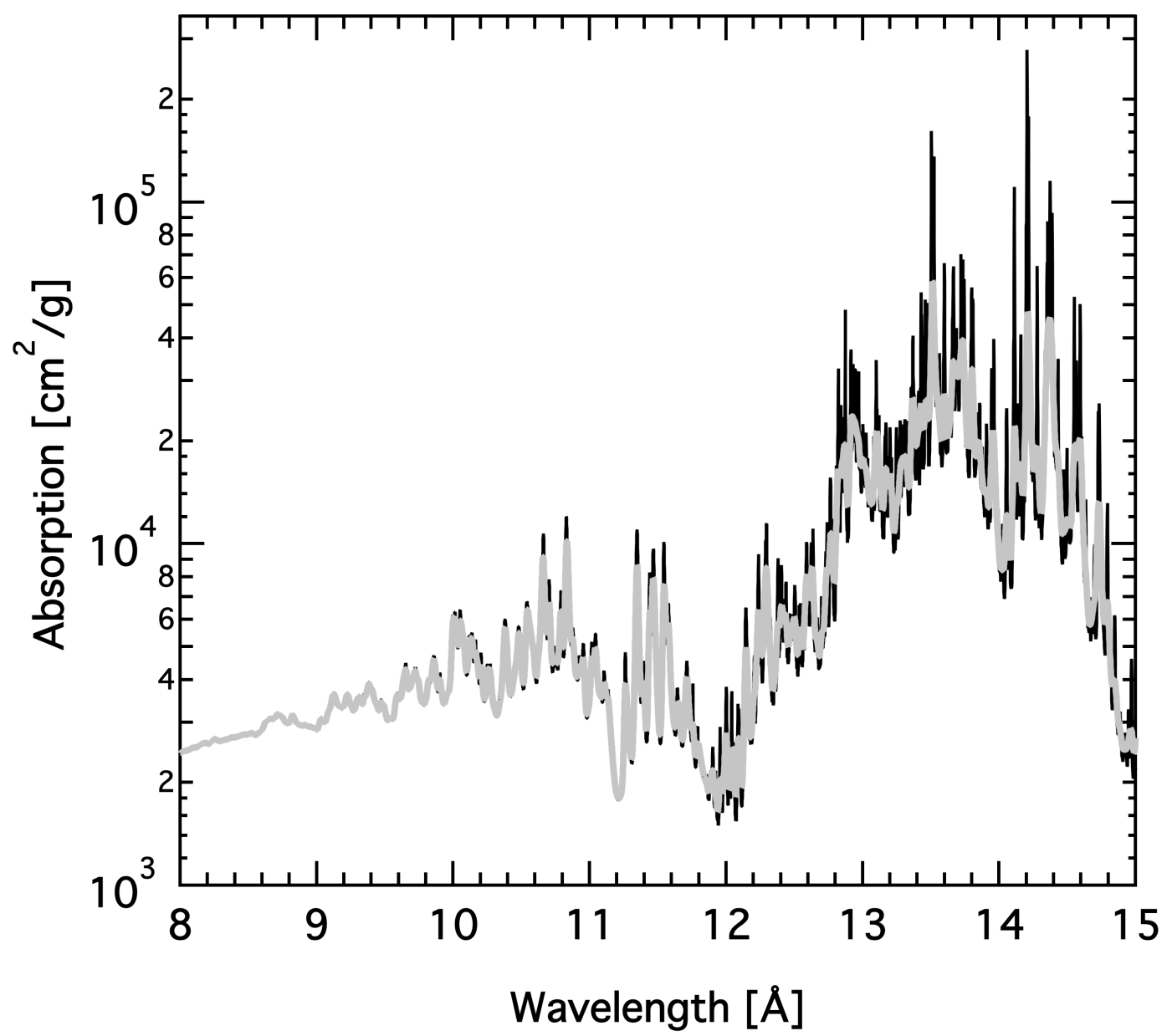

Fig 5 -Comparison of the Topaz Fe opacity (black) and effective opacity (grey) as a function of wavelength at conditions of Fig. 1. 


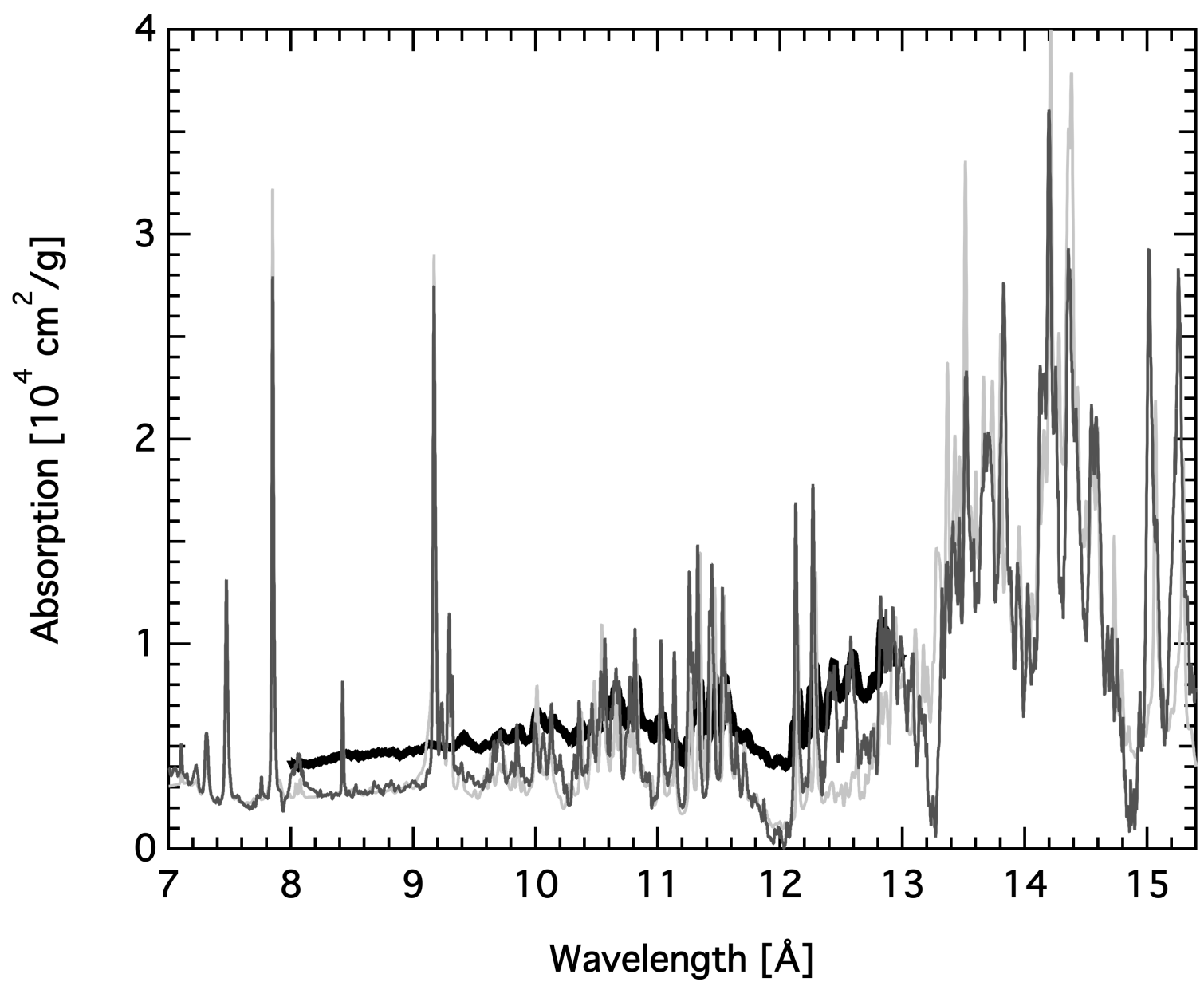

Fig 6 - Comparison of experimental and theoretical Fe photon absorption cross-section as a function of wavelength at a temperature and electron density of $156 \mathrm{eV}$ and $6.9 \times 10^{21} \mathrm{~cm}^{-}$ 3: Sandia data [2] (thin black) and Topaz effective opacity (grey). Also included is the recent Sandia data [3] (thick black) at electron temperature and density of $182 \mathrm{eV}$ and $3.1 \times 10^{22} \mathrm{~cm}^{-3}$. 


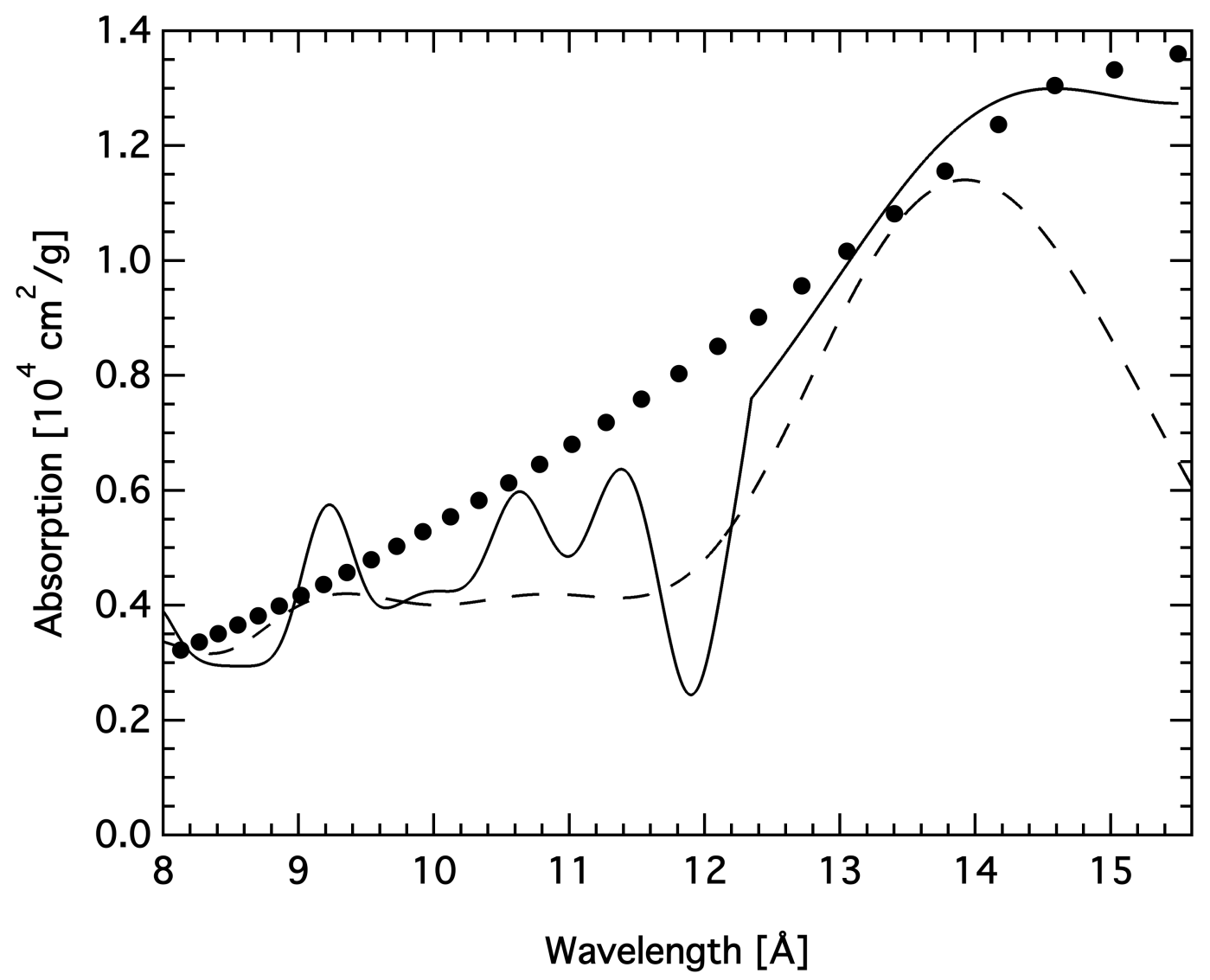

Fig 7 - The smoothed Fe photon absorption, $K(\lambda)$, as a function of wavelength at conditions of Fig. 6 for the Sandia data [2] (solid) and Topaz (dash) are compared to $\sigma_{\text {cold }}(\lambda)($ dots $)$. 